This item was submitted to Loughborough's Research Repository by the author.

Items in Figshare are protected by copyright, with all rights reserved, unless otherwise indicated.

\title{
An optimisation algorithm for designing fixed-structure controllers using the QFT method
}

PLEASE CITE THE PUBLISHED VERSION

\section{PUBLISHER}

(C) IEEE

\section{VERSION}

VoR (Version of Record)

\section{LICENCE}

CC BY-NC-ND 4.0

\section{REPOSITORY RECORD}

Nandakumar, R., G.D. Halikias, and Argyrios C. Zolotas. 2019. "An Optimisation Algorithm for Designing Fixed-structure Controllers Using the QFT Method”. figshare. https://hdl.handle.net/2134/4308. 
This item was submitted to Loughborough's Institutional Repository (https://dspace.lboro.ac.uk/) by the author and is made available under the following Creative Commons Licence conditions.

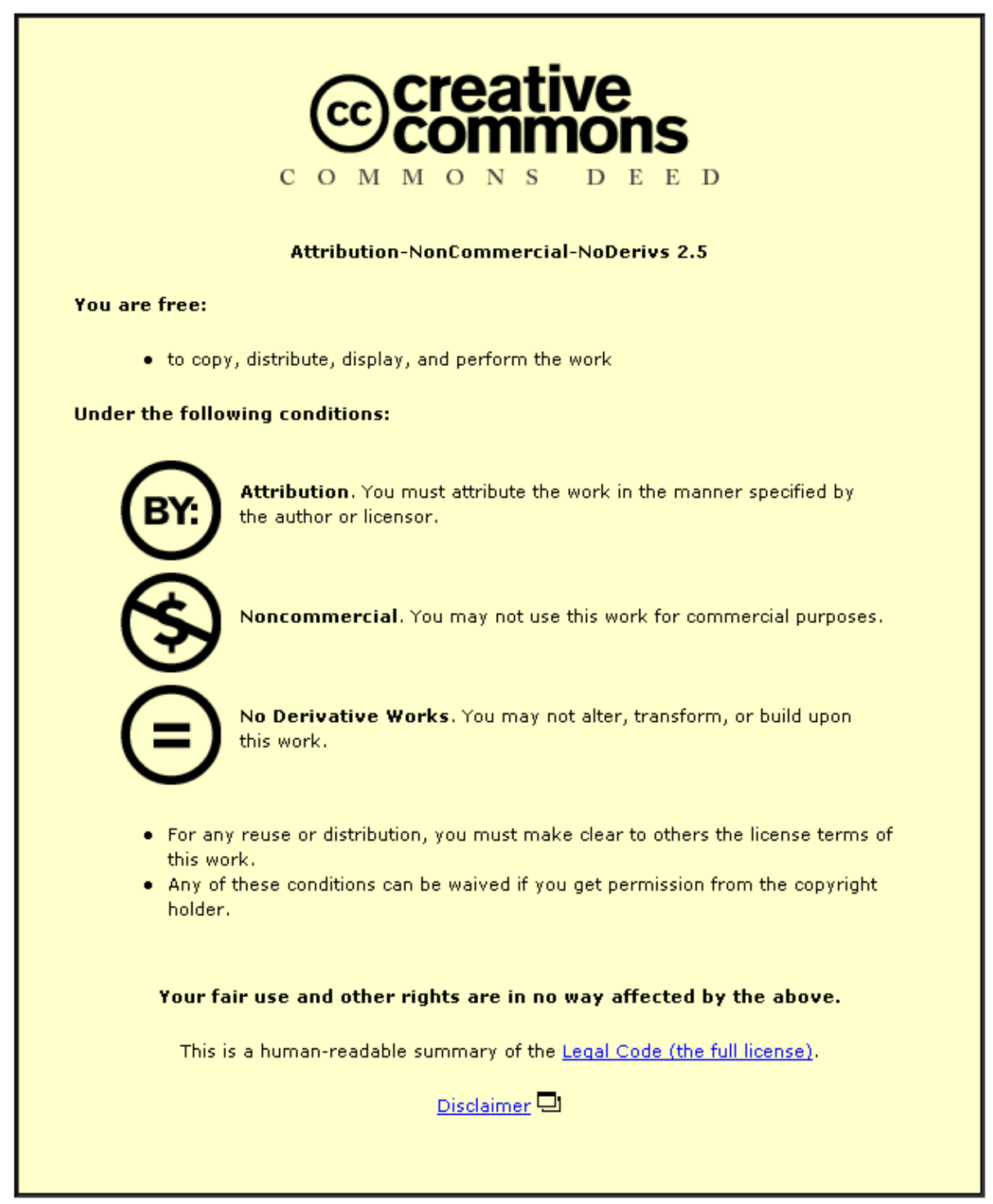

For the full text of this licence, please go to: http://creativecommons.org/licenses/by-nc-nd/2.5/ 


\section{AN OPTIMISATION ALGORITHM FOR DESIGNING FIXED-STRUCTURE CON- TROLLERS USING THE QFT METHOD}

\author{
R. Nandakumar and G.D. Halikias \\ Electronic and Electrical Engineering, \\ City University, Northampton Square, \\ London, UK, \{g.halikias@city.ac.uk, \\ r.nandakumar@city.ac.uk \}
}

Keywords: Robust Control, Quantitative Feedback Theory (QFT), Loop-shaping

\begin{abstract}
We propose a simple optimisation algorithm for designing fixed-structure controllers for highly-uncertain plants. The method can be used to automate the loop-shaping step of the QFT design procedure and guarantees robust stability and performance to the feedback loop for all parameters in the plant's uncertainty set. To avoid over-designing the system, the algorithm can be used.to minimise either the asymptotic gain or the cross-over frequency of the openloop system. Some preliminary results of this work have appeared in [9].
\end{abstract}

\section{INTRODUCTION}

Quantitative Feedback Theory is a systematic robust control design methodology for systems subject to large parametric or unstructured uncertainty. QFT is a graphical loop-shaping procedure, traditionally carried out on the Nichol's chart, which can be used for the control design of either SISO or MIMO uncertain systems, including the non-linear and time-varying cases $[3,4,8]$. Relative to other robust-control design methodologies, QFT offers a number of advantages, apart from its utilisation of classical control-design techniques. These include: (i) The ability to assess quantitatively the "cost of feedback" [5], (ii) the ability to take into account phase information in the design process (this is ignored in many norm-based approaches, e.g. $\mathcal{H}_{\infty}$ optimal control which is based on singular values), and (iii) the ability to provide "transparency" in the design, i.e. clear tradeoff criteria between controller complexity and the feasibility of the design objectives. Note that (iii) implies in practice that QFT often results in simple controllers which are easy to implement.

The QFT design procedure is based on the two-degree of freedom feedback configuration shown in Figure 1. In this diagram $G(p, s)$ denotes the uncertain plant, while $K(s)$ and $F(s)$ denote the feedback compensator and prefilter, respectively, which are to be designed. Note that model uncertainty is described by the $r$-parameter vector $p \in \mathcal{P} \subseteq \mathbb{R}^{r}$ taking values in the set $\mathcal{P}$; it is further assumed that $G(p, s)$ has the same number of RHP poles for all $p \in \mathcal{P}$. Translating the uncertainty into the frequency domain, gives rise to the plant's "uncertainty templates"

\author{
A.C. Zolotas
}

Electronic and Electrical Engineering, Imperial College, London, SW7, UK, \{a.zolotas@ieee.org\}

which are the sets:

$$
\mathcal{G}_{\omega}=\{G(p, j \omega): p \in \mathcal{P}\}
$$

For each fixed frequency $\omega, \mathcal{G}_{\omega}$ defines a "fuzzy region" on the Nichol's chart which describes the uncertainty of the plant at frequency $\omega$ in terms of magnitude (in dB's) and phase (in degrees). For design purposes, we construct $N$ uncertainty templates corresponding to a discrete set of frequencies $\left\{\omega_{1}, \omega_{2}, \ldots, \omega_{N}\right\}$ chosen to cover adequately the system's bandwidth.

The robust performance objectives of the design include good tracking of reference input $r(s)$ and good attenuation of the disturbance signal $d(s)$ entering at the system's output, despite the presence of uncertainty. The robust tracking objectives are captured by the set of inequalities:

$$
\begin{array}{r}
\max _{p \in \mathcal{P}} \Delta\left|\frac{G\left(p, j \omega_{i}\right) K\left(j \omega_{i}\right)}{1+G\left(p, j \omega_{i}\right) K\left(j \omega_{i}\right)}\right|_{\mathrm{dB}} \leq \delta\left(\omega_{i}\right) \\
:=\left.B_{u}\left(\omega_{i}\right)\right|_{\mathrm{dB}}-\left.B_{l}\left(\omega_{i}\right)\right|_{\mathrm{dB}}
\end{array}
$$

for each $i=1,2, \ldots, N$, i.e. if, for each frequency $\omega_{i}$, the maximum variation in closed loop gain as $p \in \mathcal{P}$ does not exceed the maximum allowable spread in specifications $\delta\left(\omega_{i}\right)$, typically specified via two appropriate magnitude frequency responses $B_{u}(\omega)=\left|B_{u}(j \omega)\right|$ and $B_{l}(\omega)=$ $\left|B_{l}(j \omega)\right|$. Note that it is not necessary to bound the actual gain (but only the gain spread) since we assume that, (i) no uncertainty is associated with the feedback controller $K(s)$, and (ii) the pre-filter $F(s)$ can provide arbitrary scaling to the closed-loop gain.

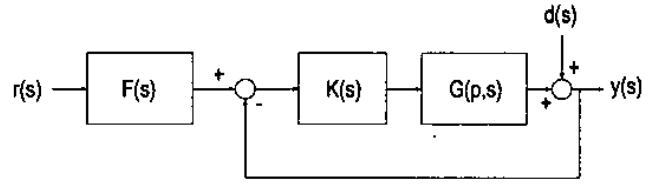

Figure 1: Feedback Configuration

The robust disturbance -rejection objective can be satisfied by bounding the sensitivity function, i.e. by imposing constraints of the form

$$
\max _{p \in \mathcal{P}}\left|\frac{1}{1+G\left(p, j \omega_{i}\right) K\left(j \omega_{i}\right)}\right| \leq D\left(\omega_{i}\right)
$$

for a (subset) of the design frequencies $\left\{\omega_{1}, \omega_{2} \ldots, \omega_{N}\right\}$. Again these are typically specified via an appropriate magnitude frequency-response $D(\omega)=|D(j \omega)|$. 
Robust stability is enforced by ensuring that: (i) no unstable pole-zero cancellations occur between the plant and the controller (for every $p \in \mathcal{P}$ ), (ii) the nominal open-loop frequency response $L_{o}(j \omega)=G\left(p_{o}, j \omega\right) K(j \omega)$ (defined for any $p_{o} \in \mathcal{P}$ ) does not cross the -1 point (i.e. the $\left(-180^{\circ}, 0\right)$ point on Nichol's chart) and makes a total number of (anti-clockwise) encirclements around it equal to the number of unstable poles of $L_{o}(s)=G\left(p_{o}, s\right) K(s)$, and (iii) That no (perturbed) open-loop response crosses the -1 point, i.e.

$$
-1 \notin \bigcup_{\omega \in \mathbf{R}} K(j \omega) \mathcal{G}_{\omega}
$$

Note that condition (i) is automatically satisfied if $K(s)$ is restricted to be stable and minimum-phase, while conditions (ii) and (iii) can be easily tested graphically $[2,1]$. In practice, a more severe condition than (iii) is imposed: To establish a minimum amount of damping, it is required that the nominal open-loop frequency response does not penetrate a closed contour in the Nichol's chart ( $\mathcal{U}$-contour); this is constructed from an appropriate $M$ circle and information about high-frequency uncertainty of the plant $[3,4]$.

The robust tracking and disturbance rejection objectives have been formulated as gain inequalities of the closed-loop transfer functions (sensitivity and complementary sensitivity) at the design frequencies. For the purposes of QFT design, these inequalities must be translated into constraints on the nominal open-loop response $L_{o}(j \omega)$. This procedure results into a number of contours ("Horowitz templates" $f_{i}^{t}(\phi)$ and "disturbance-rejection templates" $\left.f_{i}^{d}(\phi)\right)$ for each frequency $\omega_{i}, i=1,2, \ldots, N$; these are functions of the phase variable $\phi \in\left(-360^{\circ}, 0^{\circ}\right)$. Thus, robust tracking is satisfied at frequency $\omega_{i}$ if $\left|L_{o}\left(j \omega_{i}\right)\right|_{\mathrm{dB}} \geq f_{i}^{t}\left(\phi_{i}\right)$ where $\arg L_{\mathrm{o}}\left(j \omega_{i}\right)=\phi_{i}$; similarly, robust disturbance-rejection is attained at frequency $\omega_{i}$ if $\left|L_{o}\left(j \omega_{i}\right)\right|_{\mathrm{dB}} \geq f_{i}^{d}(\phi)$. The robust-performance templates (Horowitz and disturbance-rejection) can be easily constructed (within an arbitrary gain tolerance and for a discretised phase-grid) using a simple bisection algorithm.

In conclusion, assuming that the condition prohibiting unstable pole/zero cancellations between the plant and the controller is independently verified, the following conditions guarantee robust-stability and performance:

- The winding number of the nominal open-loop system $L_{o}(j \omega)$ around the -1 point is equal to the number of RHP poles of $L_{o}(s)$.

- The nominal open-loop frequency response $L_{o}(j \omega)$ does not penetrate the $\mathcal{U}$ contour.

- The following inequalities are satisfied for all $i=$ $1,2, \ldots, n$ :

$$
\left|L_{o}\left(j \omega_{i}\right)\right|_{\mathrm{dB}} \geq f_{i}^{t}\left(\phi_{i}\right)
$$

and

$$
\left|L_{o}\left(j \omega_{i}\right)\right|_{\mathrm{dB}} \geq f_{i}^{d}\left(\phi_{i}\right)
$$

in which $\phi_{i}=\arg L_{o}\left(j \omega_{i}\right)$; these inequalities correspond to the robust tracking and robust disturbancerejection specifications respectively.

\section{FORMULATION OF QFT CONSTRAINTS}

In this section we first formulate the QFT robust stability and performance constraints as a feasibility programme. This leads to an optimisation algorithm for carrying out optimal QFT designs using a family of simple fixedstructure compensators.

As was shown in the last section, the QFT robuststability and performance objectives can be translated to graphical constraints on the Nichols chart. The constraints associated with robust-performance ("Horowitz" and "disturbance-rejection" templates) correspond to open contours, i.e. they split the Nichols chart in two regions (for each design frequency), the high and low-gain region. To meet the tracking or disturbance-rejection objective, the nominal open-loop frequency response point $L_{o}\left(j \omega_{i}\right)$ must be placed on the high-gain region of the contour, i.e. forced to satisfy the inequality $\left|L_{o}\left(j \omega_{i}\right)\right|_{\mathrm{dB}} \geq f_{i}^{t}\left(\phi_{i}\right)$ (tracking) or $\left|L_{o}\left(j \omega_{i}\right)\right|_{\mathrm{dB}} \geq f_{i}^{d}\left(\phi_{i}\right)$ (disturbance rejection), where $\arg L_{o}\left(j \omega_{i}\right)=\phi_{i}$. In contrast, the robust-stability template ( $\mathcal{U}$-contour) is a closed contour containing the critical point. To construct the $\mathcal{U}$-contour, we start from the definition of an $M$-circle $(M>1)$ in the Nyquist plane $(u, v)$. After some algebra, it may be shown that the $M$ circle in the Nichols chart is a closed contour which may be decomposed into the union of the graphs of the two functions,

$$
\begin{aligned}
M^{+}(\phi) & =20 \log _{10}\left(-\cos \phi+\sqrt{\frac{1}{M^{2}}-\sin ^{2} \phi}\right) \\
& +20 \log _{10}\left(\frac{M^{2}}{M^{2}-1}\right)
\end{aligned}
$$

and

$$
\begin{aligned}
M^{-}(\phi) & =20 \log _{10}\left(-\cos \phi-\sqrt{\frac{1}{M^{2}}-\sin ^{2} \phi}\right) \\
& +20 \log _{10}\left(\frac{M^{2}}{M^{2}-1}\right)
\end{aligned}
$$

Following Horowitz [5], the $\mathcal{U}$ contour is obtained by translating $M^{-}(\phi)$ vertically by $V_{\infty} \mathrm{dB}$ 's, where

$$
V_{\infty}=\lim _{\omega \rightarrow \infty}\left(\max _{p \in \mathcal{P}}|G(p, j \omega)|_{\mathrm{dB}}-\left|G\left(p_{o}, j \omega\right)\right|_{\mathrm{dB}}\right)
$$

This gives the $\mathcal{U}$ contour as the union of the graphs of the two functions:

$$
\mathcal{U}^{+}(\phi)=M^{+}(\phi) \text { and } \mathcal{U}^{-}(\phi)=M^{-}(\phi)-\dot{V}_{\infty}
$$

over the phase interval

$$
\begin{aligned}
\phi_{l} & :=-180^{\circ}-\sin ^{-1}\left(\frac{1}{M}\right) \leq \phi \\
& \leq-180^{\circ}+\sin ^{-1}\left(\frac{1}{M}\right):=\phi_{h}
\end{aligned}
$$

The ultimate objective of this section is to characterise the regions of the Nichols chast in which the open-loop 


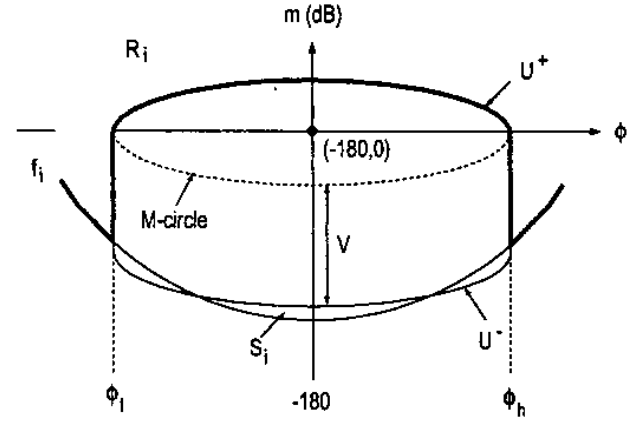

Figure 2: $M$-circle and $\mathcal{U}$-contour in Nichols chart $(M>1)$

frequency response point $L_{o}\left(j \omega_{i}\right)$ can lie in order to satisfy the robust stability and performance constraints. To this purpose define the composite function

$$
f_{i}^{m}(\phi)=\max \left\{f_{i}^{t}(\phi), f_{i}^{d}(\phi)\right\}
$$

where the maximum is taken pointwise in $\phi \in\left(-360^{\circ}, 0^{\circ}\right.$. Further define,

$$
\begin{array}{rlr}
f_{i}(\phi) & =f_{i}^{m}(\phi) & \text { for } \phi \leq \phi_{l}, \\
& =\max \left\{f_{i}^{m}(\phi), \mathcal{U}^{+}(\phi)\right\} & \text { for } \phi_{l}<\phi<\phi_{h} \\
& =f_{i}^{m}(\phi) & \text { for } \phi \geq \phi_{h}
\end{array}
$$

Also let $\Phi_{i}=\left\{\phi: \phi_{l}<\phi<\phi_{h}, f_{i}^{m}(\phi) \leq \mathcal{U}^{-}(\phi)\right\}$. Then, the robust stability and performance constraints at frequency $\omega_{i}$ are satisfied iff $L_{o}\left(j \omega_{i}\right) \in R_{i} \cup S_{i}$, where

$$
R_{i}=\left\{L_{o}\left(j \omega_{i}\right):\left|L_{o}\left(j \omega_{i}\right)\right|_{\mathrm{dB}} \geq f_{i}(\phi), \phi=\arg L_{o}\left(j \omega_{i}\right)\right\}
$$

and

$$
\begin{array}{r}
S_{i}=\left\{L_{o}\left(j \omega_{i}\right): f_{i}(\phi) \leq\left|L_{o}\left(j \omega_{i}\right)\right|_{\mathrm{dB}} \leq \mathcal{U}^{-}(\phi),\right. \\
\left.\phi=\arg L_{o}\left(j \omega_{i}\right) \in \Phi_{i}\right\}
\end{array}
$$

An illustration of the region $R_{i} \cup S_{i}$ is given in Figure 2 . Note that in practice, when a performance constraint is active, we typically have $\Phi_{i}=S_{i}=\varnothing$. This is because performance objectives are normally specified at low frequencies, rarely exceeding the closed-loop bandwidth of the system. However, our formulation allows us to take into account "unconstrained" design frequencies, i.e. frequencies at which no performance inequalities apply. For such a frequency $\omega_{i}$ we would have $f_{i}^{m}(\phi)=-\infty$ and hence $\Phi_{i}=\left(\phi_{l}, \phi_{h}\right), S_{i}=\left\{L_{o}\left(j \omega_{i}\right):\left|L_{o}\left(j \omega_{i}\right)\right|_{\mathrm{dB}} \leq \mathcal{U}^{-}(\phi), \phi=\right.$ $\left.\arg L_{o}\left(j \omega_{i}\right) \in\left(\phi_{l}, \phi_{h}\right)\right\}$ (i.e. the region below the $\mathcal{U}$ contour), while $R_{i} \cup S_{i}$ would represent the region outside the $\mathcal{U}$-contour.

The conditions that guarantee the robust-stability and robust-performance specifications at the design frequencies can now be summarised by the following two graphical tests:

- The winding number of the nominal open-loop system $L_{o}(j \omega)$ around the -1 point is equal to the number of RHP poles of $L_{o}(s)$.
- For each frequency $\omega_{i}, L_{o}\left(j \omega_{i}\right) \in R_{i} \cup S_{i}$.

Again, it is assumed that no unstable pole/zero cancellations occur between the controller and $G(p, s)$ for every $p \in \mathcal{P}$, a condition which must be checked independently. Of course, similarly to any QFT-based method, these tests do not really guarantee that $L_{o}(j \omega)$ does not enter the $\mathcal{U}$ contour (at frequencies other than the design frequencies). This, however, does not cause a problem in practice, provided a reasonably large set of design frequencies is selected near cross-over, or, alternatively, by slightly tightening the specifications by means of an appropriate tolerance.

\section{OPTIMISATION ALGORITHM}

In this section we outline an optimisation algorithm for designing fixed-structure compensators of certain types subject to the QFT constraints developed earlier. Every design (i.e. loop shaping of $L_{o}(j \omega)$ ) which satisfies the two graphical tests of the last section is in principle "admissible", i.e. satisfies the robust stability and the robustperformance objectives. Since in general many different designs may be admissible, we require a method of classifying them by formulating an appropriate optimisation criterion. Adopting the arguments of Horowitz and Sidi $[5,6]$, such a criterion must penalise the "over-design" of the system, e.g. an unnecessarily high closed-loop bandwidth, since this increases the "cost of feedback" in terms of sensor-noise amplification and potential instability due to high-frequency unmodelled dynamics/parasitics. Appropriate "cost functions" to be minimised include the following quantities: (1) Open-loop cross-over frequency (nominal or worst-case), (2) Clased-loop bandwidth (nominal or worst-case), (3) Asymptotic open-loop gain. Each of the above measures can be calculated in a straightforward manner from the frequency response of the system. Note that all these criteria depend monotonically on the system's open-loop gain.

The types of compensators considered in this paper are listed below. Note that some of these must be used under appropriate relative-degree assumptions satisfied by the transfer function of the plant.

1. PID: $K(s)=k_{p}+k_{d} s+\frac{k_{i}}{s}$

2. First-order lead/lag: $K(s)=\frac{k(s+b)}{s+a}$

3. Second-order with complex-poles (zeros): $K(s)=$ $\frac{1}{s^{2}+2 \zeta \omega_{n} s+\omega_{n}^{2}}\left(\right.$ or $\left.s^{2}+2 \zeta \omega_{n} s+\omega_{n}^{2}\right)$.

PID and phase lead/lag controllers are widely used in industry because they are simple and easy to tune. Thus optimal controllers of the first two types may provide simple solutions to robust control designs based on the QFT method. Note also that every rational controller of arbitrary complexity can be constructed from cascade interconnections of controllers in (2) and (3) above. Thus, it is possible to improve the design continuously by building higher-order controllers in a step-by-step procedure: At each step the optimisation algorithm is carried out (for one 
of the three controller structures) and the resulting optimal controller $K(s)$ is accumulated into the nominal open-loop system by redefining $L_{o}(s) \leftarrow L_{o}(s) K(s)$. This process may continue until a satisfactory design is obtained, or until the cost fails to decrease significantly. Of course, the controller resulting from this procedure will not, in general, be optimal over the higher-order controller set!

The proposed algorithm is based on the fact that fixing the phase of the compensator at two distinct frequencies determines the compensator uniquely up to scaling. Thus, the phase-response of the nominal open-loop system is also completely determined, and only a simple calculation is needed to determine the minimum amount of gain required to meet the QFT robust stability and performance specifications (if these are feasible). Geometrically, this corresponds to shifting the frequency response of $L_{o}(s)$ vertically in the Nichol's chart by the minimum gain required to place the the points $L_{o}\left(j \omega_{i}\right)$ in the $R_{i} \cup$ $S_{i}$ regions while simultaneously satisfying the Nyquist stability encirclement condition. Repeating this procedure for all possible phase combinations (suitably discretised) will eventually produce the optimal design (if one exists). Next, we consider each controller type in turn:

\subsection{PID controller:}

Theorem 1: [9] (i) Let $K(s)=k_{p}+k_{d} s+\frac{k_{s}}{s}$ with $k_{p}, k_{d}$, $k_{i}$ real parameters. Suppose that $\arg K\left(j \omega_{i}\right)=\psi_{i}$ and $\arg K\left(j \omega_{j}\right)=\psi_{j}$ where $\omega_{i} \neq \omega_{j}$. Then the matrix:

$$
A_{i j}=\left(\begin{array}{ccc}
1 & -\frac{1}{\omega_{i}^{2}} & -\frac{\tan \left(\psi_{i}\right)}{\omega_{i}} \\
1 & -\frac{1}{\omega_{j}^{2}} & -\frac{\tan \left(\psi_{j}\right)}{\omega_{j}}
\end{array}\right)
$$

has full (row) rank. Let $\left(V^{i j}\right)=\left[V_{1}^{i j} V_{2}^{i j} V_{3}^{i j}\right]^{\prime} \in \mathbb{R}^{3}$ be a (real) non-zero vector in the (one-dimensional) kernel of $A_{i j}$. Then,

$$
\left(\begin{array}{c}
k_{d} \\
k_{i} \\
k_{p}
\end{array}\right)=\lambda\left(\begin{array}{c}
V_{1}^{i j} \\
V_{2}^{i j} \\
V_{3}^{i j}
\end{array}\right)
$$

where $\lambda$ is an arbitrary real constant. Moreover, the gain and phase of the controller at any frequency $\omega$ is given by:

$$
|K(j \omega)|=|\lambda| \sqrt{\left(V_{3}^{i j}\right)^{2}+\left(V_{1}^{i j} \omega-\frac{V_{2}^{i j}}{\omega}\right)^{2}}:=|\lambda| C_{i j}(\omega)
$$

and

$$
\arg K(j \omega):=\psi(\omega)=\arctan \left(\frac{V_{1}^{i j} \omega-\frac{V_{2}^{i j}}{\omega}}{V_{3}^{i j}}\right)
$$

respectively.

(ii) If the controller gains $k_{p}, k_{i}$ and $k_{d}$ are restricted to be non-negative, then the constraints $\arg K\left(j \omega_{i}\right)=\psi_{i}$ and $\arg K\left(j \omega_{j}\right)=\psi_{j}$ are feasible if no two of the three scalars $V_{1}^{i j}, V_{2}^{i j}$ and $V_{3}^{i j}$ have opposite signs. In this case,

$\arg L_{o}\left(j \omega_{k}\right):=\phi_{k}=\arg G_{o}\left(j \omega_{k}\right)+\arctan \left(\frac{V_{1}^{i j} \omega_{k}-\frac{V_{2}^{i j}}{\omega_{k}}}{V_{3}^{i j}}\right)$
Also, $L_{o}\left(j \omega_{k}\right) \in R_{k} \cup S_{k}$ iff

- $|\lambda| \geq \frac{10^{\left(f_{k}^{m}\left(\phi_{k}\right)-\left|G_{o}\left(j \omega_{k}\right)\right|_{d \mathrm{~B}}\right) / 20}}{C_{i j}\left(\omega_{k}\right)}$ when $\phi_{k} \notin\left[\phi_{l} \phi_{h}\right]$.

- $|\lambda| \geq \frac{10^{\left(f_{k}\left(\phi_{k}\right)-\left|G_{o}\left(j \omega_{k}\right)\right|_{\mathrm{dB}}\right) / 20}}{C_{i j}\left(\omega_{k}\right)}$ when $\phi_{k} \in\left[\phi_{l} \phi_{h}\right]$ and $f_{k}^{m}\left(\phi_{k}\right) \geq M^{-}\left(\phi_{k}\right)$.

- $\frac{10^{\left(M^{-}\left(\phi_{k}\right)-\left|G_{o}\left(j \omega_{k}\right)\right|_{\mathrm{dB}}\right) / 20}}{C_{i j}\left(\omega_{k}\right)} \geq|\lambda| \geq \frac{10^{\left(f_{k}^{m}\left(\phi_{k}\right)-\left|G_{o}\left(j \omega_{k}\right)\right|_{\mathrm{dB}}\right) / 20}}{C_{i j}\left(\omega_{k}\right)}$ or $|\lambda| \geq \frac{10^{\left(M^{+}\left(\phi_{k}\right)-\left|G_{o}\left(j \omega_{k}\right)\right|_{d B}\right) / 20}}{C_{i j}\left(\omega_{k}\right)}$ when $\phi_{k} \in\left[\begin{array}{ll}\phi_{l} & \phi_{h}\end{array}\right]$ and $f_{k}^{m}\left(\phi_{k}\right)<M^{-}\left(\phi_{k}\right)$.

where $C_{i j}\left(\omega_{k}\right)$ is defined in part (i).

Theorem 1 shows that fixing the phase of the PID controller between $-90^{\circ}$ and $90^{\circ}$ at two distinct frequencies, fixes the phase of the controller at every frequency. The Nyquist plot of the PID controller (a vertical straight line with real part $k_{p}$ ) shows geometrically that in this case the three controller gains are uniquely determined (up to scaling) provided that $90^{\circ}<\psi_{i}<\psi_{j}<90^{\circ}$ for $\omega_{i}<\omega_{j}$.

The one-dimensional kernel of matrix $A_{i j}$ can be calculated efficiently using the singular value decomposition algorithm. Alternatively, the three gains can be calculated analytically in terms of the parameters $\omega_{i} \omega_{j}, \psi_{i}$ and $\psi_{j}$; this, however, does not seem to result in any significant computational advantage.

\subsection{Phase lead/lag controller}

Theorem 2: Let $K(s)=k(s+b) /(s+a)$ with $a>$ $b>0$ ("phase lead" controller). Then the constraints $\arg K\left(j \omega_{i}\right)=\psi_{i}$ and $\arg K\left(j \omega_{j}\right)=\psi_{j}$ for two distinct frequencies $\omega_{i} \neq \omega_{j}$ with $0<\psi_{i}<90^{\circ}$ and $0<\psi_{j}<90^{\circ}$ are feasible if and only if the following two conditions are satisfied:

$$
\lambda:=\frac{\left(\omega_{i}^{2}-\omega_{j}^{2}\right) \tan \psi_{i} \tan \psi_{j}}{\omega_{i} \tan \psi_{j}-\omega_{j} \tan \psi_{i}}>0
$$

and

$$
c:=\frac{\omega_{i} \omega_{j}\left(\omega_{j} \tan \psi_{j}-\omega_{i} \tan \psi_{i}\right)}{\omega_{i} \tan \psi_{j}-\omega_{j} \tan \psi_{i}}<0
$$

In this case, the quadratic equation $b^{2}+\lambda b+c=0$ has one positive root $b_{+}$and one negative root $b_{-}$and the controller parameters $b$ and $a$ are determined uniquely as $b=b_{+}$and $a=b_{+}+\lambda=-b_{-}$. In addition,

$$
\begin{aligned}
& \arg L_{o}\left(j \omega_{k}\right):=\phi_{k}=\arg G_{o}\left(j \omega_{k}\right)+\arctan \left(\frac{\omega_{k}}{b}\right)-\arctan \left(\frac{\omega_{k}}{a}\right) \\
& \text { and } L_{o}\left(j \omega_{k}\right) \in R_{k} \cup S_{k} \text { iff } \\
& \text { - } \quad k \geq \frac{10^{\left(f_{k}^{m}\left(\phi_{k}\right)-\left|G_{o}\left(j \omega_{k}\right)\right|_{\mathrm{dB}}\right) / 20}}{C\left(\omega_{k}\right)} \text { when } \phi_{k} \notin\left[\phi_{l} \phi_{h}\right] . \\
& \text { - } k \geq \frac{10^{\left(f_{k}\left(\phi_{k}\right)-\left|G_{o}\left(j \omega_{k}\right)\right| \mathrm{dB}\right) / 20}}{C\left(\omega_{k}\right)} \text { when } \phi_{k} \in\left[\phi_{l} \phi_{h}\right] \text { and } \\
& \quad f_{k}^{m}\left(\phi_{k}\right) \geq M^{-}\left(\phi_{k}\right) . \\
& \text { - } \frac{10^{\left(M^{-}\left(\phi_{k}\right)-\left|G_{o}\left(j \omega_{k}\right)\right|_{\mathrm{dB}}\right) / 20}}{C\left(\omega_{k}\right)} \geq k \geq \frac{10^{\left(f_{k}^{m}\left(\phi_{k}\right)-\left|G_{o}\left(j \omega_{k}\right)\right|_{\mathrm{dB}}\right) / 20}}{C\left(\omega_{k}\right)} \\
& \quad \text { or } k \geq \frac{10^{\left(M^{+}\left(\phi_{k}\right)-\left|G_{o}\left(j \omega_{k}\right)\right|_{\mathrm{dB}}\right) / 20}}{C\left(\omega_{k}\right)} \text { when } \phi_{k} \in\left[\phi_{l} \phi_{h}\right] \text { and } \\
& f_{k}^{m}\left(\phi_{k}\right)<M^{-}\left(\phi_{k}\right) .
\end{aligned}
$$


where $C(\omega)=\sqrt{\left(b^{2}+\omega^{2}\right) /\left(a^{2}+\omega^{2}\right)}$.

For a phase-lag controller the result is almost identical, but in this case $\lambda<0$ for constraint feasibility. Theorem 2 shows that fixing the phases of the phase lead or phase lag controller in the intervals $\left(0^{\circ}, 90^{\circ}\right)$ or $\left(-90^{\circ}, 0^{\circ}\right)$ respectively determines uniquely the dynamic part of the controller when the the constraints are feasible. Feasibility of the constraints is easily checked from two sign conditions, and the controller parameters are determined by solving a quadratic equation.

\subsection{Second-order controller with complex poles or zeros}

Theorem 3: (i) Let $K(s)=k /\left(s^{2}+2 \zeta \omega_{n} s+\omega_{n}^{2}\right)$ with $\omega_{n}>0$ and $0<\zeta<1$ ("complex-pole second-order lag"). Then the constraints $\arg K\left(j \omega_{i}\right)=\psi_{i}$ and $\arg K\left(j \omega_{j}\right)=\psi_{j}$ for two distinct frequencies $\omega_{i} \neq \omega_{j}$ with $-180^{\circ}<\psi_{i}<0^{\circ}$ and $-180^{\circ}<\psi_{j}<0^{\circ}$ and $\psi_{i} \neq \psi_{j}$ are feasible if and only if the following conditions are satisfied:

- If $\psi_{i} \neq-90^{\circ}$ and $\psi_{j} \neq-90^{\circ}$,

$$
\lambda=\frac{\omega_{j} \tan \psi_{j}-\omega_{i} \tan \psi_{i}}{\omega_{i} \tan \psi_{j}-\omega_{j} \tan \psi_{i}}>0
$$

and

$$
0<\zeta:=\frac{\tan \psi_{i}}{2}\left(\sqrt{\frac{\omega_{j} \lambda}{\omega_{i}}}-\sqrt{\frac{\omega_{i}}{\lambda \omega_{j}}}\right)<1
$$

in which case $\omega_{n}$ and $\zeta$ are defined uniquely as $\omega_{n}=\sqrt{\omega_{i} \omega_{j} \lambda}$ and via (3) respectively.

- If $\psi_{j}=-90^{\circ}$ then either of the two conditions must hold: (i) $\psi_{i} \in\left(-90^{\circ}, 0^{\circ}\right)$ and $\omega_{j}<\omega_{i}$, or (ii) $\psi_{i} \in\left(-180^{\circ},-90^{\circ}\right)$ and $\omega_{j}>\omega_{i}$, in addition to the condition

$$
\zeta:=\frac{\left(\omega_{j}^{2}-\omega_{i}^{2}\right) \tan \psi_{i}}{2 \omega_{i} \omega_{j}}<1
$$

in which case $\omega_{n}$ and $\zeta$ are uniquely determined as $\omega_{n}=\omega_{j}$ and via (4), respectively.

(ii) When the phase conditions are feasible we have

$$
\arg L_{o}\left(j \omega_{k}\right):=\phi_{k}=\arg G_{o}\left(j \omega_{k}\right)+\arctan \left(\frac{2 \zeta \omega_{n} \omega_{k}}{\omega_{n}^{2}-\omega_{k}^{2}}\right)
$$

and $L_{o}\left(j \omega_{k}\right) \in R_{k} \cup S_{k}$ iff

- $k \geq \frac{10^{\left(f_{k}^{m}\left(\phi_{k}\right)-\left|G_{o}\left(j \omega_{k}\right)\right|_{\mathrm{dB}}\right) / 20}}{C\left(\omega_{k}\right)}$ when $\phi_{k} \notin\left[\phi_{l} \phi_{h}\right]$.

- $k \geq \frac{10^{\left(f_{k}\left(\phi_{k}\right)-\left|G_{o}\left(j \omega_{k}\right)\right|_{\mathrm{dB}}\right) / 20}}{C\left(\omega_{k}\right)}$ when $\phi_{k} \in\left[\begin{array}{ll}\phi_{l} & \phi_{h}\end{array}\right]$ and $f_{k}^{m}\left(\phi_{k}\right) \geq M^{-}\left(\phi_{k}\right)$.

- $\frac{10^{\left(M^{-}\left(\phi_{k}\right)-\left|C_{o}\left(j \omega_{k}\right)\right|_{\mathrm{dB}}\right) / 20}}{C\left(\omega_{k}\right)} \geq k \geq \frac{10^{\left(f_{k}^{m}\left(\phi_{k}\right)-\left|G_{o}\left(j \omega_{k}\right)\right|_{\mathrm{dB}}\right) / 20}}{C\left(\omega_{k}\right)}$ or $k \geq \frac{10^{\left(M^{+}\left(\phi_{k}\right)-\left.1 G_{o}\left(j \omega_{k}\right)\right|_{\mathrm{dB}}\right) / 20}}{C\left(\omega_{k}\right)}$ when $\phi_{k} \in\left[\phi_{l} \phi_{h}\right]$ and $f_{k}^{m}\left(\phi_{k}\right)<M^{-1}\left(\phi_{k}\right)$. where $C(\omega)=1 / \sqrt{\left(\omega_{n}-\omega\right)^{2}+4 \zeta^{2} \omega_{n}^{2} \omega^{2}}$.

Again, Theorem 3 shows that fixing the phase of the controller at two distinct frequencies, determines completely the dynamic part of the controller, when the constraints are feasible. In the Theorem formulation the controller is restricted to be under-damped. This restriction can be removed, if required, by ignoring throughout the $\zeta<1$ condition. An almost identical procedure may be used to determine the dynamic part of a minimum-phase non-proper controller $k\left(s^{2}+2 \zeta \omega_{n} s+\omega_{n}^{2}\right)$ from its two phases in the interval $\left(0^{\circ}, 180^{\circ}\right)$ at two distinct frequencies $\omega_{i}$ and $\omega_{j}$; details are omitted.

In all three cases considered above we have derived simple gain conditions on the nominal open-loop gain, so that the QFT robust stability and performance performance constraints are satisfied. These are of the form $L_{o}\left(j \omega_{i}\right) \in R_{i} \cup S_{i}$, which for a fixed phase arg $L_{o}\left(j \omega_{i}\right)=\phi$, correspond to gain intervals

$$
\left[k_{1}\left(\omega_{i}, \phi\right), k_{2}\left(\omega_{i}, \phi\right)\right] \cup\left[k_{3}\left(\omega_{i}, \phi\right), \infty\right)
$$

where $i=1,2, \ldots, N$. Thus the optimisation problem has the form: "Minimise the optimisation criterion (i.e. cross-over frequency, closed-loop bandwidth, asymptotic open-loop gain, etc), so that for each design frequency $\omega_{i}, i=1,2, \ldots, N, L_{o}\left(j \omega_{i}\right) \in R_{i} \cup S_{i}$ and Nyquist's encirclement criterion is satisfied". Since for the three types of controllers described above the phase of the nominal open-loop system is completely determined once two controller phases have been fixed, we can use the following algorithm for solving the optimisation problem:

\section{Optimisation algorithm:}

1. Obtain a phase array $\Phi$ by discretising the phase interval $\left(-360^{\circ} 0^{\circ}\right]$.

2. Select any two distinct frequencies $\omega_{i}$ and $\omega_{j}$ in the set of design frequencies $\left(\omega_{1}, \omega_{2}, \ldots, \omega_{N}\right)$.

3. Calculate the phase intervals $\Phi_{k} \subseteq \Phi$ and $\Phi_{l} \subseteq \Phi$ in which the nominal open loop phase $\arg L_{o}(j \omega)$ can vary at $\omega=\omega_{k}$ and $\omega=\omega_{l}$ respectively. These depend on the type of controller to be designed, e.g. for a PID controller they lie within $\pm 90^{\circ}$ of $\arg G\left(p_{o}, \omega_{k}\right)$ and $\arg G\left(p_{o}, \omega_{l}\right)$, etc.

4. Initialise an $m \times n$ array $F$ where $m$ and $n$ are the sizes of $\Phi_{k}$ and $\Phi_{l}$ respectively, to contain the value of the objective function (cross-over frequency, closedloop bandwidth, asymptotic gain etc.) for each phase pair. Also, initialise three $m \times n$ controller parameter arrays to contain the parameters $\left(k_{p}, k_{d}, k_{i}\right)$ for a PID controller, $\left(k_{o}, a, b\right)$ for a phase-lead/lag controller, or $\left(k_{o}, \omega_{n}, \zeta\right)$ for a second-order controller with complex poles/zeros.

5. For each $\left(\Phi_{k}(i), \Phi_{l}(j)\right) \in \Phi_{k} \times \Phi_{l} \subseteq \mathbb{R}^{m \times n}$ :

(a) Calculate $\psi_{i}=\Phi_{k}(i)-\arg G\left(p_{o}, \omega_{k}\right)$ and $\psi_{j}=$ $\Phi_{l}(j)-\arg G\left(p_{o}, \omega_{l}\right)$ 
(b) Determine a controller $K(s)$ of one of the three types discussed earlier, such that $\arg K\left(j \omega_{k}\right)=$ $\psi_{i}$ and $\arg K\left(j \omega_{l}\right)=\psi_{j}$. If these phase constraints are infeasible, set $F(i, j)=\infty$ and consider the next phase pair $\left(\Phi_{k}(i), \Phi_{l}(j)\right)$.

(c) Find the minimum value of gain $k_{o}>0$ such that (i) $L_{o}\left(j \omega_{q}\right)=k_{o} K\left(j \omega_{q}\right) G\left(p_{o}, j \omega_{q}\right) \in R_{q} \cup$ $S_{q}$ for all $q=1,2, \ldots, N$ and (ii) $L_{o}(j \omega)$ satisfies Nyquist's encirclement criterion. If no such gain $k_{o}$ exists, set $F(i, j)=\infty$ and consider the next phase pair $\left(\Phi_{k}(i), \Phi_{l}(j)\right)$.

(d) Calculate the value of the objective function (cross-over frequency, closed-loop bandwidth, asymptotic gain, etc) corresponding to the designed $L_{o}(j \omega)$ and assign it to the $(i, j)$ th element of $F$. Save also the controller parameters to the corresponding entries of the parameter arrays.

6. Calculate $\gamma_{0}=\min _{(i, j) \in \Phi_{k} \times \Phi_{l}}(F)$ and $\left(i^{*}, j^{*}\right) \in$ $\operatorname{argmin}(F)$. If $\gamma_{0}=\infty$ the QFT constraints are infeasible; otherwise the optimal cost is $\gamma_{o}$ and the optimal controller parameters can be obtained from the $\left(i^{*}, j^{*}\right)$ th elements of the controller-parameter arrays.

A few remarks on the algorithm:

- In step (1) of the algorithm the phase discretisation of the interval $\left(-360^{\circ}, 0^{\circ}\right)$ results in a phase grid $\Phi$, typically equally spaced. In practice, $50-100$ phases are adequate. It is helpful to calculate the performance bounds ("Horowitz and "disturbancerejection" templates) over the same phase grid.

- In principle any two frequencies $\omega_{k}$ and $\omega_{l}$ can be selected from the set of design frequencies in step (2). Selecting these frequencies reasonably far-apart (for minimum numerical sensitivity) works well in practice.

- In steps (3) and (5a) of the algorithm all phase calculations can be performed modulo $-360^{\circ}$. This restricts the phase interval of interest to the range $\left(-360^{\circ}, 0^{\circ}\right]$.

- Since the phase of $L_{0}(j \omega)$ is completely determined when two controller phases are fixed, the calculation of the minimum gain in step (5c) is straightforward. Checking the total number of encirclements required for stability is also straightforward and can be performed by purely graphical means (i.e. by counting the crossings of the $-180^{\circ}$ line and their directions). See $[2,1]$ for details. Note also, that a frequency grid "denser" than the set of design frequencies must be used for this purpose.

- Step 5(c) requires the calculations of the performance bounds at arbitrary phases, which may not coincide with the discretised phases of array $\Phi$. There is no difficulty, however, in estimating the performance gains from adjacent phase points, e.g. using linear interpolation. Alternatively, the performance bounds may be calculated exactly at these phases to arbitrary accuracy using a bisection algorithm implemented between steps $(5 \mathrm{~b})$ and $(5 \mathrm{c})$.

\section{EXAMPLE}

Due to lack of space it is not possible to include an example in this paper. A number of numerical examples illustrating the method will be included at the conference presentation.

\section{CONCLUSIONS}

An algorithm for the control design of highly-uncertain systems has been outlined. The algorithm is simple, easy to implement, and can be used to automate the loop-shaping step of the QFT design procedure. It can be used to design robust-performance optimal controllers of a simple structure (PID, phase-lead/lag, second-order), or more complex controllers involving arbitrary interconnections of these structures. Extensions of the method to multivariable systems is possible using the standard QFT approach $[7,8]$.

\section{REFERENCES}

[1] W. Chen and D. J. Ballance. Stability analysis on the Nichols chart and its application in QFT.- Preprint, University of Glasgow, August 1997.

[2] W. Chen and D. J. Ballance. QFT design for uncertain non-minimum phase and unstable plants. Preprint, University of Glasgow, July 1997.

[3] J. D'Azzo and C. Houpis. Feedback control systems analysis and synthesis. Prentice Hall, 1998.

[4] I. M. Horowitz. Synthesis of Linear Systems. Academic Press; 1973.

[5] I. M. Horowitz and M. Sidi. Synthesis of linear systems with large plant ignorance for prescribed time-domain tolerances. Int. J. Control, 16:287-309, 1972.

[6] I. M. Horowitz and M. Sidi. Optimum synthesis of nonminimum phase systems with plant uncertainty. Int. $J$. Control, 27:361-386, 1978.

[7] J. M. Maciejowski. Multivariable feedback design. Addison-Wesley, 1989

[8] O. Yaniv and I. M. Horowitz. A quantitative design method for MIMO linear feedback systems having uncertain plants. Int. J. Control, 43:401-421, 1986.

[9] A. C. Zolotas and G. D. Halikias. Optimal design of PID controllers using the QFT method. IEE Proceedings - Control Theory Appl., 146(6), November 1999. 